Cockcroft-11-11

\title{
Using Monolithic Active Pixel Sensors for Fast Monitoring of Therapeutic Hadron Beams
}

\author{
R. Boll ${ }^{\mathrm{a}}$, M. Caccia ${ }^{\mathrm{b}}$, C.P. Welsch ${ }^{\mathrm{c}}$, M.H. Holzscheiter ${ }^{\mathrm{a}, \mathrm{d}}$
}

${ }^{a}$ Max-Planck-Institute for Nuclear Physics, Heidelberg, Germany.

'Universit`a dell’ Insubria, Como, Italy.

${ }^{c}$ Cockcroft Institute and University of Liverpool, UK.

${ }^{\mathrm{d}}$ University of New Mexico, Albuquerque NM, USA 


\section{Accepted Manuscript}

Title: Using Monolithic Active Pixel Sensors for Fast Monitoring of Therapeutic Hadron Beams

Authors: R. Boll, M. Caccia, C.P. Welsch, M.H. Holzscheiter

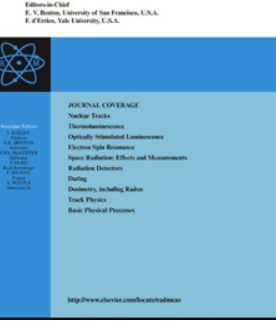

PII:

$$
\text { S1350-4487(11)00220-4 }
$$

DOI:

Reference: $\quad$ RM 4439

To appear in: Radiation Measurements

Please cite this article as: Boll, R., Caccia, M., Welsch, C.P., Holzscheiter, M.H. Using Monolithic Active Pixel Sensors for Fast Monitoring of Therapeutic Hadron Beams, Radiation Measurements (2011), doi: 10.1016/j.radmeas.2011.05.053

This is a PDF file of an unedited manuscript that has been accepted for publication. As a service to our customers we are providing this early version of the manuscript. The manuscript will undergo copyediting, typesetting, and review of the resulting proof before it is published in its final form. Please note that during the production process errors may be discovered which could affect the content, and all legal disclaimers that apply to the journal pertain. 


\title{
Using Monolithic Active Pixel Sensors for Fast Monitoring of Therapeutic Hadron Beams
}

\author{
R. Bolla , M. Caccia ${ }^{\mathrm{b}}$, C.P. Welsch ${ }^{\mathrm{c}}$, M.H. Holzscheiter ${ }^{\mathrm{a}, \mathrm{d}}$ \\ ${ }^{a}$ Max-Planck-Institute for Nuclear Physics, Heidelberg, Germany. \\ ${ }^{b}$ Università dell' Insubria, Como, Italy. \\ ${ }^{c}$ Cockcroft Institute and University of Liverpool, UK. \\ ${ }^{d}$ University of New Mexico, Albuquerque NM, USA.
}

\begin{abstract}
It has been shown that Monolithic Active Pixel Sensors (MAPS) are very promising tools for direct online beam monitoring, for current heavy ion therapy facilities as well as for future innovative cancer treatments with antiprotons. More specific, the dead-time free Mimotera sensor has been proven to be capable of dealing with extremly short pulses of antiprotons of only $500 \mathrm{~ns}$ duration, as well as with continuous beams of carbon ions in the complete intensity- and energy range used in today's heavy ion facilities. It shows a linear behavior up to $7.5 \times 10^{7}$ antiprotons per square centimeter in $500 \mathrm{~ns}$ and up to $8 \times 10^{8}$ carbon ions per square centimeter per second .
\end{abstract}

Keywords:

Monolithic Active Pixel Sensor, Mimotera, Beam monitor, Cancer therapy, Antiprotons

\section{Introduction and Motivation}

Nowadays, tumors are most frequently treated by irradiation with X-rays. Anyhow, in the last 60 years a new technology has been developed that is especially beneficial for deep seated tumors and which is in clinical use since 1990 [1]: The treatment of cancer with hadron beams, most commonly with protons or carbon ions. From a biological point of view, heavy ions have one outstanding advantage over photons, namely the mechanism of energy loss along their path through the body, resulting in a sharp rise of the energy deposition at the very end of their track, called the Bragg-peak. The idea of using antiprotons for cancer therapy, however, is rather new [2] and there is only one experiment worldwide doing research on this: The Antiproton Cell Experiment (ACE) [3], located at the AntiprotonDecelerator (AD) of the European Organization for Nuclear Research (CERN).

The physical behavior of antiprotons is very similar to protons, with one crucial difference: Once the antiproton annihilates with a proton at the end of its range, it releases an additional energy of $2 \times 936 \mathrm{MeV}=1.87 \mathrm{GeV}$ per particle as compared to protons, of which a small but clinically significant fraction is deposited locally. This means that to achieve the same energy transfer to the tumor in the Bragg-peak region, half as many particles are needed and the dose in the entrance channel in front of the tumor is reduced, sparing healthy tissue and organs at risk in the beam path, possibly overcoming detrimental effects due to a low dose isotropic background from antiproton annihilation.

The experimental set-up at ACE is as follows: The antiproton beam enters a Poly(methyl methacrylate)(PMMA)tank which contains a probe of V-79 Chinese Hamster cells embedded into gelatin. For reasons of cooling it is surrounded by a mixture of water and glycol, that also simulates the back scattering from a real biological target. After the irradiation, cell survival curves are obtained from evaluating the probes. As the biological effect of particles on living cells is the quantity to be evaluated, it is crucial to know as precisely as possible the physical characteristics 
of the incident beam as the input parameters for all following analyses. The properties necessary for the evaluation of the data are the diameter and the shape of the beam profile, the fluctuations of the beam intensity from shot to shot and the beam position.

The situation at ACE is unique in the sense of the spill structure: The beam of antiprotons is pulsed, one spill is $500 \mathrm{~ns}$ long and arrives only every $90 \mathrm{~s}$ due to the accelerator architecture. During these $500 \mathrm{~ns}$ about $3 \times 10^{7}$ particles are delivered to an area of $0.4 \mathrm{~cm}^{2}$. This leads to a momentary intensity of $3 \times 10^{7} /\left(500 \mathrm{~ns} \times 0.4 \mathrm{~cm}^{2} \times \pi\right)=$ $1.19 \times 10^{14} \mathrm{~s}^{-1} \mathrm{~cm}^{-2}$. The beam energy was chosen as $126 \mathrm{MeV}$ for a $12 \mathrm{~cm}$ penetration depth in water.

For this special situation a detector is required that is capable of handling this amount of particles in such a short time. It must not saturate in order to be able to get the true number of particles for each pixel and it must provide a fast, digital output for every single shot, which can then immediately be used for correcting the beam, if necessary. Previous systems such as ionization chambers, gafchromic films or scintillating screens were lacking at least one of these abilities. This gave rise to the search for a new device, the most promising candidate being a Monolithic Active Pixel Sensor (MAPS).

\section{Architecture of the sensor}

The Mimotera (Minimum Ionizing MOnolithic active pixel sensor (MAPS) for the TERA foundation) is a MAPS of crystalline silicon. The epitaxial layer is only $14 \mu \mathrm{m}$ thick and has an entrance window of about $100 \mathrm{~nm}$. [4] This is possible by thinning the wafer down and back-illuminating it. The sensor consists of $112 \times 112=12544$ pixels, each of the size of $153 \times 153 \mu \mathrm{m}^{2}$. This leads to an active area of $17 \times 17 \mathrm{~mm}^{2}$, which is divided into four sub-arrays of $28 \times 112$ pixels that are read out in parallel. There are two rows and two columns per sub-quarter which act as so called "dummy-pixels". These are not physical pixels, so there is no dead area on the sensor, but they are introduced to allow matching the one-dimensional, linear analog output with the two-dimensional matrix in the data analysis. The clockrate for the read-out for a single pixel can be set in LabView as $2.5,5,10$, and $20 \mathrm{MHz}$. At the maximum setting the readout of one sub-array therefore takes $50 \mathrm{~ns} \times(112+2) \times(28+2)=170 \mu \mathrm{s}$. As the four sub-arrays are read out in parallel, this is identical to the readout time for one frame of the whole detector. In the following measurements a clock rate of $2.5 \mathrm{MHz}$ per pixel has been chosen, which leads to an integration time of $1.4 \mathrm{~ms}$.

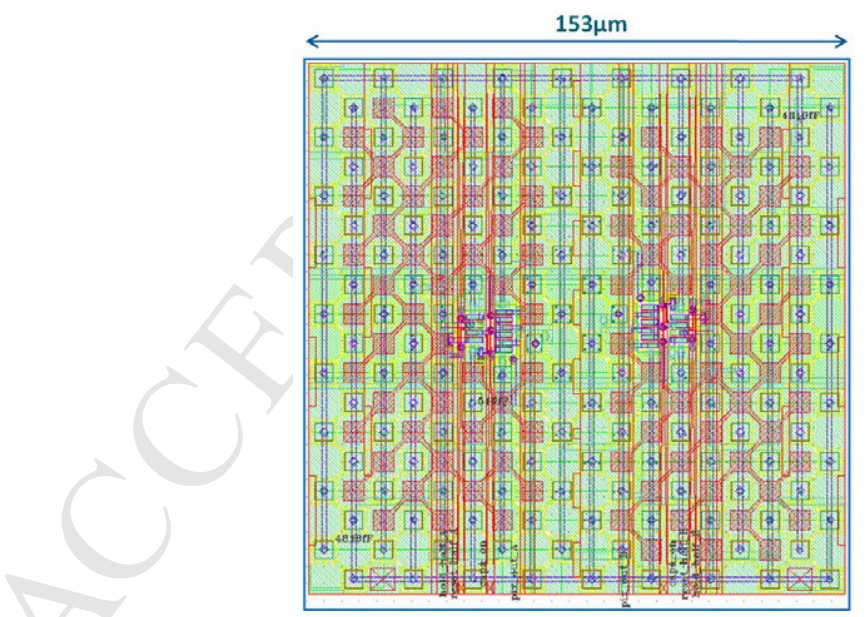

Figure 1: Design of one of the pixels of the Mimotera. All red and all yellow diodes are connected, building two completely independent, interleaved readout matrices for each of the pixels. (Courtesy of M. Caccia)

The design of one single pixel makes the Mimotera a unique device: Each of the pixels consists of $2 \times 81$ diodes, $5 \times 5 \mu \mathrm{m}^{2}$ each, corresponding to the red and yellow squares in Figure 1, building two independent readout matrices which will in the following be referred to as matrix A and matrix B. This architecture makes the Mimotera a detector without any dead time: While matrix A is collecting the generated charge carriers, the charges stored in the diodes of 
matrix B are read out, the diodes are reset, afterwards the process is inverted. Hence, no signal charges get lost during the readout and the detector is free of dead time.

\section{Measurements at CERN}

In June 2010 first tests have been carried out at ACE. The beam spill of $500 \mathrm{~ns}$ duration is contained in only one readout frame of $1.4 \mathrm{~ms}$ of the sensor. Therefore it is most convenient to analyze the data in a differential way, subtracting the frame with the spill from the previous one without beam. This has the advantage that one does not have to record pedestal files. All dead pixels and time-dependent background signals are eliminated automatically.

The crucial question for the first test run was to find out whether the detector is really capable of coping with the high momentary intensity of ACE. Investigations into possible saturation effects in the beam core were carried out. If such effects were present, they would limit the detector's capability of measuring the full beam intensity and determining the beam profile with high precision.
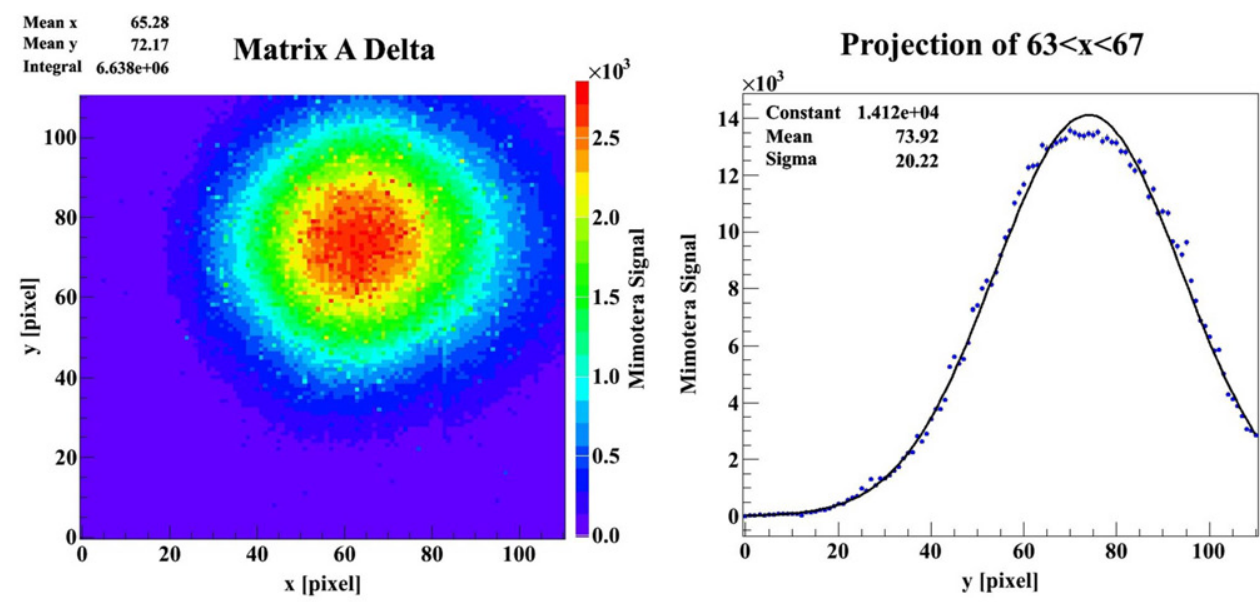

Figure 2: Profile of one shot of antiprotons in the Mimotera. Mean values in x- and y-direction are displayed, as well as the integral over the whole detector surface. The right picture includes a Gaussian fit.

Figure 2 shows the profile of one single beam spill in the Mimotera. The data matches well with the Gaussian fit displayed on the projection. Reference measurements using gafchromic films resulted in a Gaussian beam shape of the same sigma value. The linearity of the detector can also be tested by comparing the integrated signal in the Mimotera to the nominal beam intensity obtained from the accelerator log file. A deviation from linearity of less than $1 \%$ has been observed, caused by saturation visible at the highest intensities in the center of the beam in Figure 2 [5].

\section{Measurements at HIT}

The Mimotera has not only been tested with antiprotons, but also with carbon ions at the Heidelberg Ion-Beam Therapy Center (HIT) in Heidelberg, Germany. The aim of these measurements was to investigate whether it could serve as a beam monitor for existing cancer treatment facilities. A scan through all available beam intensities from $5.0 \times 10^{6} \mathrm{~s}^{-1}$ to $8.0 \times 10^{7} \mathrm{~s}^{-1}$ has been performed, the results are presented in Figure 3 .

The detector response is very good, clear beam spots were obtained from the lowest to the highest intensity. The behavior is linear within the uncertainties of the nominal beam intensity provided by the accelerator operation software. These measurements allowed concluding that the Mimotera is suitable for direct monitoring of carbon ion beams used in current heavy ion facilities.

It is, however, not applicable for monitoring proton beams at HIT at the moment, because the energy deposition per particle is too small for a good signal at the available beam intensities. The threshold of the Mimotera is about 
Carbon ions I7

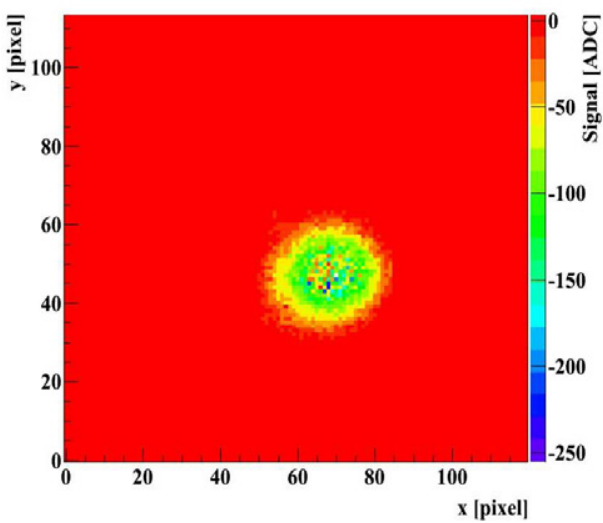

Linearity of Mimotera signal with the beam intensity

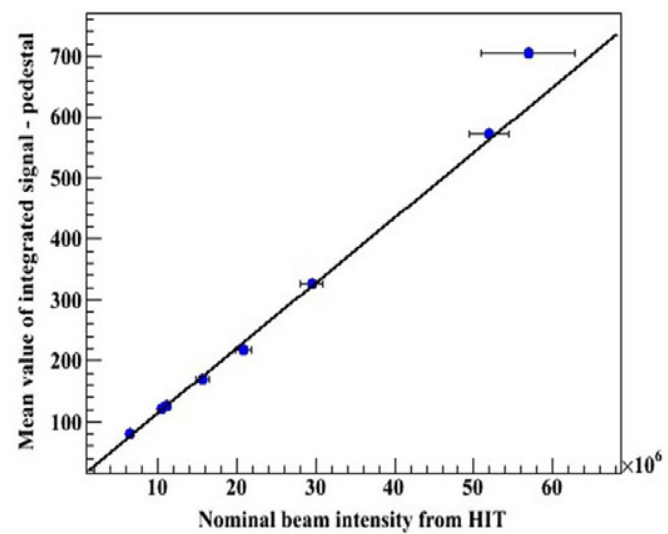

Figure 3: On the left: Profile of carbon beam integrated over two minutes. On the right: Linearity plot obtained from the intensity scan. The signal is averaged over the whole spill and background is subtracted.

5000 electrons per pixel per frame which corresponds to an energy deposit of $5000 \times 3.62 \mathrm{eV}=18.1 \mathrm{keV}$ in silicon. Carbon ions of $410 \mathrm{MeV} / \mathrm{n}$ deposit $288 \mathrm{keV}$ in $14 \mu \mathrm{m}$ of silicon, in contrast to protons of $211 \mathrm{MeV}$ which deposit only $12 \mathrm{keV}$. [6] Lower proton energies with higher ionization unfortunately could not be tested at HIT, because the focus size of the beam increases rapidly with decreasing energy, e.g. $32 \mathrm{~mm}$ minimum focus for $50 \mathrm{MeV}$ protons.

\section{Summary and Outlook}

It has been shown that the Mimotera is well suited for direct monitoring of very short pulses of antiprotons as well as of continuous beams of carbon ions. Further investigation will focus on monitoring proton beams. To be able to monitor even larger focus sizes, one could merge four of the sensors together. To reduce the beam straggling further, such that the sensor could stay in the beam during treatment, it is possible to remove that part of the printed circuit board support which lies behind the active area of the detector.

\section{Acknowledgments}

The Mimotera architecture is protected by the U.S. patent no. 7582875 , it was developed by the SUCIMA collaboration, with a project supported by the European Commission under the contract no. G1RD-CT-2001-00561. This work was supported by the DFG under contract WE3565-3 and the NSF under grant \# CBET 0853157. MHH acknowledges support by the EU through a Marie Curie Fellowship under contract \# PIIF-GA-2009-234814. Special thanks go to M. Caccia at Università dell' Insubria, Como, for providing the detector and for much technical and scientific support, and to L. Negrini for being a great help in the data analysis.

\section{References}

[1] http://ptcog.web/psi.ch/

[2] T. E. Kalogeropoulos, R. Muratore; Nucl. Inst. Meth. B40/41 (1989) 1322-25.

[3] M. Holzscheiter et al.; Radiotherapy \& Oncology, vol. 81, (2006) pp. 233-242.

[4] L. Bandano: Developpement d'un moniteur de faisceau innovant pour la mesure en temps reel des faisceaux utilises en hadron therapie. $\mathrm{PhD}$ thesis, 2005

[5] R. Boll: A Monolithic Active Pixel Sensor as Direct Monitor for Therapeutic Antiproton and Ion Beams Diploma thesis, 2010. Publication in refereed journal in preparation.

[6] http://www.sgeier.net/tools/bbt.html 